\title{
Interstyloid Distances in Northeastern Thailand
}

\author{
Distancias Interestiloides en el Noreste de Tailandia
}

\author{
Wiwanitkit, V.
}

WIWANITKIT, V. Interstyloid distances in Northeastern Thailand. Int. J. Morphol., 35(2):393-393, 2017.

Dear Editor, i read the recent publication by Waraporn et al., on "Sexual Dimorphism Using the Interstyloid Distances and Clinical Implication for Elongated Styloid Process in Northeastern Thailand" with a great interest (Sakaew et al., 2016). Waraporn et al., noted that "it is suggested that the styloid process can be applied to the sex identification by measuring the interstyloid distance at the base or the tip of these processes" (Sakaew et al.). There is an important note on the forgotten possible confounding factor for this report. The study is from Northeastern Thailand where the prevalence of thalassemia and hemoglobinopathy is extremely high. Waraporn et al., did not exclude the cases with underling thalassemia and hemoglobinopathy. Anyone with thalassemia and hemoglobinopathy can have the bone problem (including the maxilla) (Di Matteo et al., 2008) and this can be the missed uncontrolled confounding factor in the study by Waraporn et al.

WIWANITKIT, V. Distancias en interestiloides en el Noreste de Tailandia. Int. J. Morphol., 35(2):393-393, 2017.

He leído con gran interés la reciente publicación de Waraporn et al., titulada "El Dimorfismo Sexual Utilizando las Distancias Interestiloides y la Implicación Clínica para el Proceso Estiloides Elongado en el Noreste de Tailandia" (Sakaew et al., 2016). Waraporn et al. señalan "se sugiere que el proceso estiloideo puede aplicarse a la identificación del sexo mediante la medición de la distancia interestiloide en la base o en la punta de estos procesos" (Sakaew et al.). Hay una nota importante sobre el factor de confusión posiblemente olvidado en este informe. El estudio es del Noreste de Tailandia, donde la prevalencia de talasemia y hemoglobinopatía es extremadamente alta. Waraporn et al. no excluyeron los casos con talasemia subyacente y hemoglobinopatía. Cualquier persona con talasemia y hemoglobinopatía puede tener patologías del hueso (incluyendo el hueso maxilar) (Di Matteo et al., 2008) y esto puede ser el factor de confusión omitido en el estudio de Waraporn et al.

\section{REFERENCES}

Sakaew, W.; Arnanteerakul, T.; Somintara, S.; Ratanasuwon, S.: Uabundit, N.; Iamsaard, S.; Chaisiwamongkol, K.; Chaichun, A. \& Hipkaeo, W. Sexual dimorphism using the interstyloid distances and clinicalimplication for elongated styloid process in Northeastern Thailand. Int. J. Morphol., 34(4):1223-7, 2016.

Di Matteo, R.; Liuzza, F.; Manicone, P. F.; Raffaelli, L.; Berardi, D.; Perfetti, G. \& Maccauro, G. Bone and maxillofacial abnormalities in thalassemia: a review of the literature. J. Biol. Regul. Homeost. Agents, 22(4):211-6, 2008.

Corresponding author:

Professor Viroj Wiwanitkit

Wiwaniitkit House, Bangkhae

Bangkok

THAILAND

E-mail: wviroj@yahoo.com

Received: 06-02-2017

Accepted: 22-02-2017 